\title{
High-grain feeding causes strong shifts in ruminal epithelial bacterial community and expression of Toll-like receptor genes in goats
}

\author{
Jun-hua Liu, Gao-rui Bian, Wei-yun Zhu and Sheng-yong Mao* \\ Department of Animal Nutrition and Feed Science, Laboratory of Gastrointestinal Microbiology, College of Animal Science and Technology, Nanjing Agricultural \\ University, Nanjing, China
}

\section{Edited by:}

Lee Mark Wetzler, Boston University

School of Medicine, USA

\section{Reviewed by:}

Xun Suo, China Agricultural

University, China

Qendrim Zebeli, University of

Veterinary Medicine Vienna, Austria

*Correspondence:

Sheng-yong Mao, Department of

Animal Nutrition and Feed Science, Laboratory of Gastrointestinal

Microbiology, College of Animal

Science and Technology, Nanjing

Agricultural University, Weigang No.

1, Nanjing 210095, China

e-mail: maoshengyong@163.com
High-grain (HG) feeding used in intensive goat production can affect the physiology of the rumen wall, but the changes induced in the epimural bacterial community and host Toll-like receptors (TLRs) are not well understood. In this study, 10 male goats were randomly allocated to two groups and fed either a hay diet $(0 \%$ grain; $n=5)$ or an HG diet $(65 \%$ grain; $n=5)$. The changes in the ruminal epithelial bacterial community and expression of TLRs during long-term (7 weeks) HG feeding were determined using pyrosequencing and quantitative real-time polymerase chain reaction. Principal coordinate analysis and analysis of molecular variance (AMOVA) results showed that HG feeding caused a strong shift in bacterial composition and structure. At the genus level, our data revealed that it increased the relative abundance of taxa Butyrivibrio, unclassified Clostridiales, Mogibacterium, unclassified Anaerolineaceae, and Succiniclasticum, and decreased the proportion of unclassified Ruminococcaceae, unclassified Rikenellaceae, unclassified Erysipelotrichaceae, Howardella, and unclassified Neisseriaceae. The HG-fed goats also exhibited upregulation of the relative mRNA expression of TLR2, TLR3, and TLR5 in the rumen epithelium $(P<0.05)$. Correlation analysis revealed that the increase in TLR expression was associated with changes in the relative abundance of ruminal epithelial bacteria. This study provides a first insight into the adaptive response of ruminal epithelial bacterial populations to HG feeding in goats and shows that these changes were associated with alterations in TLR expression. These findings provide new insight into understanding of host-microbial relationships in ruminants.

Keywords: high-grain feeding, bacterial community, ruminal epithelium, Toll-like receptors, goat

\section{INTRODUCTION}

In current intensive goat production, to meet the energy demand for fast-growing goats, high-grain (HG) diet feeding has become common practice in the nutritional management of meat goats in China. It is well known that HG diet feeding affects ruminal fermentation characteristics and the structure of the contentassociated rumen microbial population (Russell and Rychlik, 2001; Callaway et al., 2010; Hook et al., 2011; Metzler-Zebeli et al., 2013; Petri et al., 2013; Zened et al., 2013). However, similar information regarding ruminal epithelial (epimural) bacteria is incomplete compared to knowledge of the bacterial community in rumen content.

Through the use of electron microscopy and culturedependent (McCowan et al., 1978, 1980; Cheng and Wallace, 1979) and culture-independent (Cheng et al., 1980; Sadet et al., 2007; Li et al., 2012) techniques, it has been found that epimural bacteria are different from those associated with rumen contents in dairy cattle, steer, and sheep. The epimural bacterial community has some specific functions, such as hydrolysis of urea, scavenging of oxygen, and recycling of epithelial tissue (Cheng and Wallace, 1979; Wallace et al., 1979; Dinsdale et al., 1980; Petri et al., 2013). Some studies have demonstrated, using $16 S$ rRNA sequencing, polymerase chain reaction and denaturing gradient gel electrophoresis (PCR-DGGE), and quantitative reverse transcription PCR (qRT-PCR) analysis (Sadet-Bourgeteau et al., 2010; Chen et al., 2011), that ruminal epithelial bacterial population and diversity can be affected by dietary changes. Nevertheless, information on epimural bacterial community numbers has been limited, due to the low throughput of the traditional 16S rRNA clone library method and fingerprint profiles. Determining $16 \mathrm{~S}$ rRNA short variable tags using a high-throughput sequencing technology such as 454 pyrosequencing has provided an unprecedented sequencing depth, with tens to thousands of reads per sample. As a result, there has been renewed interest in measuring and comparing the composition and richness of microbial taxa in epimural samples in ruminant. However, only one published report to date has investigated changes in epimural bacterial richness and diversity in HG-fed heifers using high-throughput pyrosequencing techniques (Petri et al., 2013). In addition, little is known about the changes in epimural bacterial community of meat goats during HG diet feeding, especially depending on the high-throughput technique.

Toll-like receptors (TLRs) make up a group of pattern recognition receptors that are commonly identified in the gastrointestinal 
tissues of monogastric animals (Abreu, 2010; Malmuthuge et al., 2012). Recently, Malmuthuge et al. (2012) reported that TLRs110 can express in the rumen epithelium of calves. It is well known that TLRs can recognize the sensing of host epimural bacteria and bacterial products and trigger an immune response, which is critical for maintaining host-microbial homeostasis in monogastric animals (Hooper et al., 2012). Dysregulation of this process can cause chronic inflammatory and epithelial barrier dysfunction (Abreu, 2010; Ulluwishewa et al., 2011). Our previous study demonstrated that HG diet feeding caused massive barrier dysfunction and local inflammation in the rumen epithelium (Liu et al., 2013). If results from monogastric intestinal tissues are applied to the rumen epithelium, it seems that HG-induced rumen epithelial barrier dysfunction is associated with dysregulation of host TLR signaling. Furthermore, the changes in rumen epithelial TLR expression might be linked to the host epimural bacterial community (Chen et al., 2012). Thus, in the present study, we hypothesized that (1) HG feeding can cause an alteration in epimural bacterial community and (2) such a change might be associated with variation in the rumen epithelial TLRs expression. Therefore, the objective of this study was to investigate changes in the richness and diversity of epimural bacterial communities and the expression of host TLR genes during HG feeding in goats.

\section{MATERIALS AND METHODS}

The experimental design and procedures were approved by the Animal Care and Use Committee of Nanjing Agricultural University, in compliance with the Regulations for the Administration of Affairs Concerning Experimental Animals (The State Science and Technology Commission of P. R. China, 1988).

\section{ANIMALS, DIETS, AND EXPERIMENTAL DESIGN}

This animal experiment was carried out at the experimental station of Nanjing Agricultural University in Jiangsu Province, China. The experimental design was previously described in detail (Liu et al., 2013). Briefly, ten rumen-cannulated, castrated male goats (Boer $\times$ Yangtze River Delta White) aged 2-3 years were used in this experiment. Prior to the experiment, all of the goats were fed pure hay ad libitum for 5 weeks to ensure adaptation to the low-energy hay diet. The goats were then randomly assigned to groups fed either a hay diet (Hay; $n=5$ ) or an HG diet (HG; $n=5)$, and placed in individual pens $(1.2 \times 1.2 \mathrm{~m})$ with free access to water. Adaptation to the HG diet was carried out over $5 \mathrm{~d}$ with progressively increasing amounts of grain for $65 \%$. The diet (750 g dry matter per animal per day) was offered in equal amounts at 08:30 and 16:30 daily for 7 weeks. Hay fed in this experiment originated from one batch. The metabolic energy citation(ME) intake of the goats in the hay group ( $30 \mathrm{~kg}$ body weight; $8.31 \mathrm{MJ} / \mathrm{kg}$ dry matter) was slightly above the requirement for maintenance, and that of the goats in the HG group (30 kg body weight; $11.31 \mathrm{MJ} / \mathrm{kg}$ dry matter) permitted a growth rate of $200 \mathrm{~g} /$ day according to guidelines on the nutrient requirements of goats (NY/Y816-2004; Ministry of Agriculture of China, 2004). The nutrient compositions of the hay and HG diets are summarized in Supplementary Table 1. No significant differences in body weight gain $(0.30 \pm 0.26$ vs. $1.02 \pm 0.52 \mathrm{~kg}, P=0.248)$ or dry matter intake were observed between the hay and HG groups during the feeding trial.

\section{SAMPLE COLLECTION}

On day 50, 4-5 h after the last feeding according to the normal continuous feeding protocol, the goats were euthanized by captive bolt stunning followed by exsanguination from the carotid arteries. Within $5 \mathrm{~min}$, a segment of the rumen epithelial tissue was collected and immediately washed three times in ice-cold phosphate-buffered saline. The samples were divided into two portions. The first portion of the tissue sample was cut into smaller pieces (approximately $0.5 \times 0.5 \mathrm{~cm}$ ) and immediately frozen in liquid nitrogen for RNA extraction. The second tissue sample portion was cut to approximately $2 \times 2 \mathrm{~cm}$ and scraped from the underlying tissue using a germ-free glass slide, immediately transferred into liquid $\mathrm{N}$, and then stored at $-80^{\circ} \mathrm{C}$ until DNA extraction. Washed rumen papillae were immediately fixed in $2.5 \%$ glutaraldehyde for microscopic analysis.

\section{MORPHOMETRIC ANALYSIS}

Fixed rumen papillae was prepared for electron microscopy using methodology reported by Graham and Simmons (2005). Samples were then examined using scanning electron microscopy (Hitachi Model S-3000N, Hitachi Technologies, Tokyo, Japan) and transmission electron microscopy (Hitachi H-7650, Hitachi Technologies, Tokyo, Japan).

\section{REAL-TIME QUANTITATIVE PCR (qPCR)}

Total RNA was extracted from the rumen epithelium tissue with acid using TRIzol (Takara Bio, Otsu, Japan), as described by Chomczynski and Sacchi (1987). The RNA concentration was then quantified using a NanoDrop spectrophotometer (ND1000UV-Vis; Thermo Fisher Scientific, Waltham, MA). The absorption ratio $(260 / 280 \mathrm{~nm})$ of all of the samples was between 1.8 and 2.0, indicating high RNA purity. Aliquots of RNA samples were subjected to electrophoresis through a $1.4 \%$ agaroseformaldehyde gel to verify integrity. The concentration of RNA was adjusted to $1 \mu \mathrm{g} / \mu \mathrm{l}$ based on optical density and stored at $-80^{\circ} \mathrm{C}$. Total RNA $(1 \mu \mathrm{g})$ was reverse-transcribed using a PrimeScript ${ }^{\circledR}$ RT Reagent Kit with gDNA Eraser (Takara Bio) according to the manufacturer's instructions.

The primers used for TLR2 (forward - 5'-CTGTGTGCGTC TTCCTCAGA- $3^{\prime}$ and reverse - $5^{\prime}$-TCAGGGAGCAGAGTAACCA GA-3'), TLR3 (forward - 5'-TCTTTTCGGGACTGTTGACC$3^{\prime}$ and reverse - $5^{\prime}$-AAATCCCCCATCCAAGGTAG-3'), TLR4 (forward - 5'-GGTTTCCACAAAAGCCGTAA- $3^{\prime}$ and reverse - $5^{\prime}$-AGGACGATGAAGATGATGCC-3'), and TLR5 (forward - 5'-TCAATGGGAGCCAGATTTTC-3' $3^{\prime}$ and reverse $5^{\prime}$-CCTTCAGCTCCTGGAGTGTC- $3^{\prime}$ ) were described by Charavaryamath et al. (2011), and the primer used for GAPDH (forward - 5'-GGGTCATCATCTCTGCACCT- $3^{\prime}$ and reverse 5'-GGTCATAAGTCCCTCCACGA-3') was described by Wang et al. (2009). All of the primers were synthesized by Invitrogen Life Technologies (Shanghai, China). Real-time qPCR of the target genes and GAPDH was performed using an ABI 7300 real-time PCR system (Applied Biosystems, Foster City, CA) 
with fluorescence detection of SYBR green dye. Amplification conditions were as follows: $95^{\circ} \mathrm{C}$ for $30 \mathrm{~s}$ followed by 40 cycles of $5 \mathrm{~s}$ at $95^{\circ} \mathrm{C}$ and $31 \mathrm{~s}$ at $57.5^{\circ} \mathrm{C}$ (for GAPDH) or $62^{\circ} \mathrm{C}$ (for the TLRs). Each sample contained 1-10 ng cDNA in $2 \times$ SYBRGreen PCR Master Mix (Takara Bio) and $200 \mathrm{nmol} / \mathrm{L}$ of each primer in a final volume of $20 \mu \mathrm{L}$. All measurements were performed in triplicate. A reverse-transcription-negative blank of each sample and a no-template blank served as negative controls. The relative amount of each studied mRNA was normalized to GAPDH mRNA levels as a housekeeping gene, and the data were analyzed according to the $2^{-\triangle \Delta \mathrm{CT}}$ method.

\section{MICROBIAL DNA ISOLATION}

One gram of underlying rumen epithelial tissue was used for DNA extraction. The DNA was extracted by a bead-beating method using a mini-bead beater (Biospec Products, Bartlesville, OK), followed by phenol-chloroform extraction (Sun et al., 2008). The solution was precipitated with ethanol, and the pellets were suspended in $50 \mu \mathrm{L}$ Tris-EDTA buffer. The DNA samples were quantified using a NanoDrop spectrophotometer (Nyxor Biotech, Paris, France).

\section{DNA PYROSEOUENCING}

Bacterial amplicons were sequenced using the 454 GS FLX Titanium chemistry at the Majorbio Bio-Pharm Technology Co., Ltd., Shanghai, China. PCR amplification of the V1-V3 region of bacterial $16 \mathrm{~S}$ rRNA was performed using universal primers (27F 5'-AGAGTTTGATCCTGGCTCAG-3', 533R 5'TTACCGCGGCTGCTGGCAC-3') incorporating FLX Titanium adapters and a sample barcode sequence. The primers were synthesized by Invitrogen Life Technologies (Shanghai, China). The PCR amplification was conducted in a $50 \mu \mathrm{L}$ C1000 thermal cycler (Bio-Rad, USA) containing $10 \mu \mathrm{L} 5$-fold reaction buffer, $50 \mathrm{ng}$ of total genomic DNA, $0.4 \mu \mathrm{M}$ of each primer, $0.5 \mathrm{U}$ Pfu polymerase (TransStart-FastPfu DNA Polymerase, TransGen Biotech, Beijing, China) and 2.5 mMdNTPs. The cycling parameters were as follows: $5 \mathrm{~min}$ initial denaturation at $95^{\circ} \mathrm{C} ; 25$ cycles of denaturation at $95^{\circ} \mathrm{C}(30 \mathrm{~s})$, annealing at $55^{\circ} \mathrm{C}(30 \mathrm{~s})$, elongation at $72^{\circ} \mathrm{C}(30 \mathrm{~s})$; and final extension at $72^{\circ} \mathrm{C}$ for 5 min. Three separate PCR reactions of each sample were pooled for pyrosequencing. Amplicons from the two microbial groups were quantified fluorometrically, normalized per sample and pooled per microbial group. A total of $1 \mu \mathrm{g}$ DNA of each of the four resulting pools was loaded onto agarose gel ( $1 \%$ wt: vol). Bands were visualized and excised under blue light transillumination, and amplicons were gel purified using a QIAquick Gel Extraction Kit (Qiagen, Hilden, Germany). Amplicons were then quantified using a Quant-iT PicoGreen dsDNA Assay Kit (Invitrogen, Carlsbad, CA) according to the manufacturer's instructions. Equal concentrations of amplicons were pooled from each sample. Emulsion PCR of pooled samples was done with the GS FLX Titanium Lib-L LV emPCR Kit (Roche Applied Science, Indiananpolis, IN) following the manufacturer's recommendations.

\section{PYROSEQUENCING DATA ANALYSIS}

The sequences were processed using the MOTHUR program (Schloss et al., 2009, 2011; version 1.29.0; University of Michigan; http://www.mothur.org/wiki/). 16S rRNA reads were decoded based on the $5 \mathrm{bp}$ sample-specific barcodes and processed to remove poor-quality sequences. To reduce sequencing errors, the shhh.flows command was applied, which is the MOTHUR implementation of the AmpliconNoise algorithm (Quince et al., 2011). Quality filters were applied to trim and remove sequences with the following characteristics: less than $200 \mathrm{bp}$ in length; average quality score less than 35 ; homopolymers longer than eight nucleotides; and more than two different bases to the primer. In order to obtain a non-redundant set of sequences, unique sequences were determined, and used to align against the SILVA reference alignment database (Pruesse et al., 2007); chimeras were removed using chimera.uchime (http://drive5. com/uchime); sequences identified as being of eukaryotic origin were removed; the candidate sequences were screened and preclustered to eliminate outliers; and a distance matrix was generated from the resulting sequences. Sequences were clustered into operational taxonomic units (OTUs) using the furthest neighbor algorithm. Representative sequences from OTUs at a 0.03 distance were obtained and classified using the Ribosomal Database Project's Bayesian classifier (Wang et al., 2007). Rarefaction curve was generated at the level of $3 \%$, which was calculated by the distance-based OTU (Schloss et al., 2011), and Rarefaction curves and Good's coverage were calculated to quantify the coverage and sampling effort. Community diversity was estimated with the unnormalized reads using the based coverage estimator (ACE), Chao1, and Shannon indices. The unweighted UniFrac distance method (Lozupone and Knight, 2005) was used to perform a principal coordinates analysis (PCOA) with all OTUs, and a distance-based analysis of molecular variance (AMOVA) was conducted to assess significant differences between samples.

The $16 \mathrm{~S}$ sequencing data for all the samples analyzed in this study was submitted to the Sequence Read Archive (SRA; http:// www.ncbi.nlm.nih.gov/Traces/sra/), under accession SRP052769.

\section{STATISTICAL ANALYSIS}

Statistical analysis were carried out by conducting tests using the SPSS software package (SPSS version 16, SPSS, Inc.). The normality of the distribution of variables was tested using the Shapiro-Wilk test. The microbial diversity data, some data of taxa richness, and the relative mRNA expression of TLRs and cytokines found to have a normal distribution were analyzed by the independent-samples $t$-test procedure, according to the following model: $\mathrm{Y}=\mu+\mathrm{C}+\mathrm{e}$, where $\mu$ is the mean, $\mathrm{C}$ is the effect of diet, and e is the residual error. The Kruskal-Wallis test was used to analyze variables found to have a non-normal distribution (some data of taxa richness), and the statistical model used: $\mathrm{H}=\frac{12}{n(n+1)} \sum_{i=1}^{k} \frac{R i^{2}}{n_{i}}-3(n+1)$ where $\mathrm{H}$ is the KruskalWallis test; $n$ is the number of measurements; $R_{i}$ is the sum of the ranks; $n_{i}$ is the number of experiments. Significance was declared at $P<0.05$.

Double dendrograms were constructed using the comparative functions and multivariate hierarchical clustering methods of NCSS 2007 (NCSS), on the basis of the abundance of bacterial groups at different taxonomic levels. Clustering with all OTUs was performed using the Ward's minimum variance method with no scaling. Correlations between the relative mRNA expression 
of TLRs and the relative abundance of epimural bacteria and cytokine mRNA expression were assessed by Pearson's correlation test $\left(\gamma=\frac{\sum X Y-\frac{\left(\sum X\right)-\left(\sum Y\right)}{n}}{\sqrt{\left(\sum X^{2}-\frac{\left(\sum X\right)^{2}}{n}\right)\left(\sum Y^{2}-\frac{\left(\sum Y\right)^{2}}{n}\right)}}\right)$, where $\gamma$ is the Pearson correlation coefficient; $\mathrm{X}$ is the relative mRNA expression of TLRs; $\mathrm{Y}$ is the epimural bacteria data found to have a normal distribution or cytokine mRNA expression; $n$ is the number of experiments) or Spearman's rank test $\left(\gamma=1-\frac{6 \sum d^{2}}{n^{3}-n}\right.$, where $\gamma$ is Spearman's Rank correlation coefficient; $d$ is the difference in rank between the relative mRNA expression of TLRs and the epimural bacteria data found to have a non-normal distribution, $n$ is the number of measurements) depending on data normality using GraphPad Prism version 5.00 (GraphPad Software, San Diego, CA). Significance was declared at $P<0.05$.

\section{RESULTS}

The body weight gain $(0.30 \pm 0.26$ vs. $1.02 \pm 0.52 \mathrm{~kg}, P=0.248)$ or dry matter intake were not affected by experimental diet during the feeding trial.

\section{RUMINAL pH AND CONCENTRATIONS OF VOLATILE FATTY ACIDS, LACTATE, AND LIPOPOLYSACCHARIDES}

The data of rumen fermentation of experimental goats were reported in our previous study (Liu et al., 2013) (Supplementary Table 2). Briefly, HG feeding decreased $(P<0.001)$ the ruminal $\mathrm{pH}$, and increased $(P<0.001$ to $<0.019)$ the concentrations of propionate, butyrate, valerate, isovalerate, total volatile fatty acids, lactate, and lipopolysaccharides significantly.

\section{EPIMURAL BACTERIAL COMMUNITY OF GOATS}

In total, 55,633 reads were obtained for the $16 \mathrm{~S}$ rRNA genes in rumen epithelium of all goats, and 38013 reads were valid correspondingly, accounting for $68.3 \%$ of their raw reads. The sequences were further analyzed by MOTHUR software, and these valid sequences were classified into 7673 OUTs at sequence divergences of 0.03 . The average number of OTUs was $1364 \pm$ 138 , with an average coverage of $85.25 \pm 1.72 \%$. The Chaol richness, ACE, and Shannon diversity indexes were on $2953 \pm$ $360,4764 \pm 660$, and $6.02 \pm 0.13$, respectively. Within the bacterial population, 18 phyla were found across all samples. Firmicutes, Bacteroidetes, and Proteobacteria, were the dominant phyla, representing $53.12 \%, 20.88 \%$, and $13.35 \%$, respectively. Actinobacteria, Chloroflexi, and Tenericutes represented average percentages of $2.79 \%, 2.25 \%$, and $1.32 \%$, respectively, of the total sequences. The proportion of some phyla (Spirochaetes, Fibrobacteres, Synergistetes, and Planctomycetes) was less than 1\% of total microbial community, and other phyla (Lentisphaerae, Elusimicrobia, Chlamydiae, Cyanobacteria, Deinococcus-Thermus, Fusobacteria, and Nitrospirae) were not consistently present in all of the different host populations. In the Firmicutes phylum, the most dominant class was Clostridia (average of $48.45 \%$, respectively, of the total sequences), represented mainly by families Lachnospiraceae, unclassified Clostridiales, and Ruminococcaceae (average of $27.14 \%, 10.48 \%$, and $8.09 \%$, respectively, of the total sequences). In the Bacteroidetes phylum, the most dominant class was Bacteroidia (average of $20.38 \%$, respectively, of the total sequences), represented mainly by families Prevotellaceae,
Rikenellaceae, and unclassified Bacteroidales (average of 10.92\%, $4.69 \%$, and $4.51 \%$, respectively, of the total sequences). In the Proteobacteria phylum, the major classes were Deltaproteobacteria and Betaproteobacteria (average of $5.74 \%$ and $5.35 \%$, respectively, of the total sequences), represented mainly by families Desulfobulbaceae, Comamonadaceae, and Neisseriaceae (average of $5.51 \%, 2.90 \%$, and $2.36 \%$, respectively, of the total sequences). At the genus level, 100 taxa were detected over the entirety of the samples; the most dominant genus was Butyrivibrio (average of $11.01 \%$ of the total sequences), followed by the genera Desulfobulbus (5.51\%), Mogibacterium (4.33\%), and Prevotella $(4.01 \%)$. For clarity and visualization purposes, the top 30 bacterial taxa are presented in a heat map (Supplementary Figure 1).

\section{EFFECT OF HG DIET ON EPIMURAL BACTERIAL DIVERSITY AND RELATIVE ABUNDANCE LEVELS}

The rarefaction curves of epimural bacterial communities are shown in Supplementary Figure 2. At dissimilarity levels of 0.03, the rarefaction analysis revealed that HG feeding decreased bacterial diversity in the rumen epithelium compared with the hay group. The unweighted UniFrac metric in MOTHUR was used to evaluate $\beta$-diversity across the samples (Figure 1). The PCOA result exhibited that the goats fed the hay diet were distinctly separated from those fed the HG diet in the plot (Figure 1A; axis $1+$ axis $2=37.3 \%)$. The further analysis indicated that the diet significantly affected the epimural microbial communities (AMOVA, Fs $=2.75, P<0.001$ ). Mean pairwise unweighted UniFrac distance showed that there was no significant difference in sample-to-sample variation among replicates between the hay and HG groups (Figure 1B; $0.63 \pm 0.01$ vs. $0.62 \pm 0.01$; $P=0.482$ ). The hay vs. $H G$ comparison variation was greater than the sample-to-sample variation among replicates in the hay group (Figure 1B; $0.73 \pm 0.01$ vs. $0.63 \pm 0.01 ; P<0.001$ ) and the HG group (Figure 1B; $0.73 \pm 0.01$ vs. $0.62 \pm 0.01 ; P=0.482$ ). In addition, the Venn profile revealed that there were 299 unique OTUs belonging to the hay-fed goats and 227 unique OTUs belonging to the HG-fed goats (Supplementary Figure 3).

As is shown in Table 1, the results indicated a decrease in OTU numbers $(P=0.016)$, ACE $(P=0.047)$, and Chaol richness $(P=0.028)$, but not in community evenness [Shannon's $(P=0.117)$ and Simpson's $(P=0.465)]$ during HG diet feeding. At the phylum level, HG feeding caused a higher proportion of Firmicutes $(P=0.008)$ and Chloroflexi $(P=0.008)$ and a negative effect on Actinobacteria $(P=0.008)$ and Tenericutes $(P=$ $0.008)$ phyla; the Bacteroidetes and Proteobacteria phyla were not affected significantly $(P>0.05)$ (Supplementary Figure 4$)$. At the genus level, a total of 100 taxa were examined, 24 of which exhibited significant variability across diets. There was a significant increase in relative abundance of dominant genera Butyrivibrio $(P=0.008)$, unclassified Clostridiales $(P=0.008)$, Mogibacterium $(P=0.008)$, unclassified Anaerolineaceae $(P=$ $0.008)$, Succiniclasticum $(P=0.008)$, and Ruminococcus $(P=$ 0.008 ) associated with $\mathrm{HG}$ feeding, and the proportion of Butyrivibrio was almost three times higher in the HG group than in the hay group (Table 2). Conversely, there was a corresponding decrease in the proportion of unclassified Ruminococcaceae $(P=0.008)$, unclassified Rikenellaceae $(P=0.008)$, unclassified 

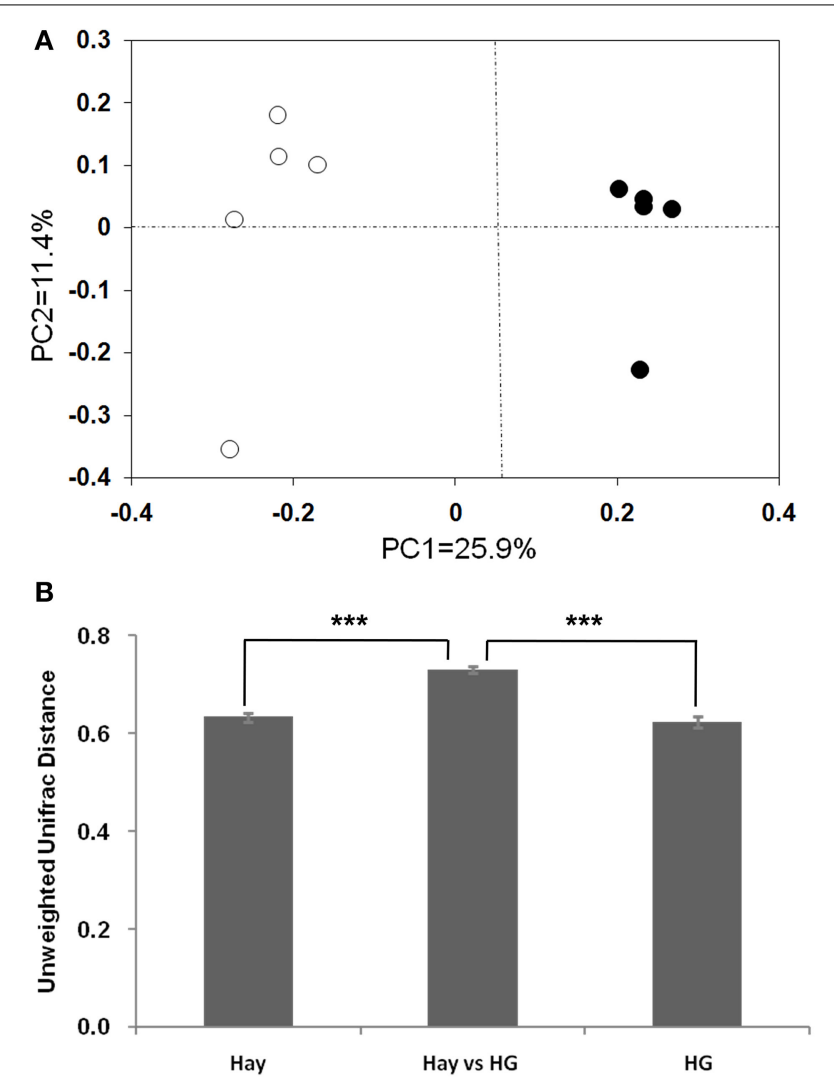

FIGURE 1 | Differences in the ruminal epithelial (epimural) bacterial structure between hay and high-grain (HG)-fed goats. (A) Unweighted UniFrac principal coordinate analysis (PCoA) of epimural microbiota based on the operational taxonomic unit data from 454 pyrosequencing run. The marks relate to the diets of donor goats: hay diet (o), HG diet (•). (B) Mean pairwise unweighted UniFrac distance for subsets of samples (means \pm SEM, $n=5$ ). Hay, sample-to-sample variation among replicates in hay group; HG, sample-to-sample variation among replicates in HG group; Hay vs. HG, hay vs. HG comparison variation. ${ }^{* *} P<0.001$

Table 1 | Effects of high-grain (HG) diet feeding on the diversity of ruminal epithelial bacterial community at the $3 \%$ dissimilarity level.

\begin{tabular}{lccccc}
\hline OTU & ACE & $\begin{array}{c}\text { Chao } \\
\text { 1 value }\end{array}$ & $\begin{array}{c}\text { Shannon } \\
\text { index }\end{array}$ & Evenness \\
\hline Hay & $1615 \pm 227$ & $5821 \pm 1069$ & $3561 \pm 599$ & $6.24 \pm 0.23$ & $0.77 \pm 0.01$ \\
HG & $1113 \pm 56$ & $3708 \pm 507$ & $2345 \pm 200$ & $5.79 \pm 0.05$ & $0.75 \pm 0.00$ \\
$P$-value & 0.016 & 0.047 & 0.028 & 0.117 & 0.602 \\
\hline
\end{tabular}

Values shown are means $\pm S E M, n=5$.

OTU, operational taxonomic units; ACE, abundance-based coverage estimator.

Erysipelotrichaceae $(P=0.008)$, Howardella $(P=0.008)$, and unclassified Neisseriaceae $(P=0.008)$ (Table 2). All of the analysis results showed that HG feeding had a considerable effect on epimural bacterial composition and diversity.

\section{CORE EPIMURAL BACTERIAL COMMUNITY}

The OTU detected across all samples was defined as the core epimural bacterial community. The results indicated that
$16.9 \%$ of the OTUs appeared in all samples belonging to Butyrivibrio (6.47\% of total sequences), Desulfobulbus $(3.84 \%$ of total sequences), unclassified Erysipelotrichaceae (2.91\% of total sequences), unclassified Prevotellaceae (2.90\% of total sequences), Comamonas (2.45\% of total sequences), and Howardella $(2.26 \%$ of total sequences). We also compared the unique OTUs associated with hay and HG diet. In the hay group, $47.2 \%$ of the OTUs were found to be unique to this diet. Unclassified Neisseriaceae was found to be $(4.48 \%$ of total sequences) unique in the goats fed the hay diet, but it was not found in HG-fed goats. During the HG diet feeding, $35.9 \%$ of the OTUs were found to be unique to this diet: unclassified Lachnospiraceae ( $4.56 \%$ of total sequences), unclassified Clostridiales (4.19\% of total sequences), and Butyrivibrio (3.97\% of total sequences). Genus Ruminococcus ( $1.32 \%$ of total sequences) was found to be unique to the HG-fed goats.

\section{EFFECTS OF HG FEEDING ON RELATIVE mRNA EXPRESSION OF TLR AND CYTOKINE GENES}

The relative mRNA expression of TLR genes, measured by qRTPCR, are shown in Figure 2. The results revealed that the HG-fed goats indicated upregulation of the relative mRNA expression of TLR2 $(P=0.005)$, TLR3 $(P=0.004)$, and TLR5 $(P=0.001)$ in rumen epithelia compared with the hay-fed goats. HG feeding had no significant effect on the relative mRNA expression of TLR4 $(P=0.089)$. The relative expression of cytokine in rumen epithelium were reported in our previous study (Liu et al., 2013). Briefly, the data exhibited that the relative expression of TNF- $\alpha$ $(P=0.017)$ and IFN- $\gamma(P=0.012)$ of HG-fed goats were higher than that of hay-fed goats significantly, and HG feeding had no significant effect on the relative expression of IL-1 $\beta(P=0.066)$, IL-6 $(P=0.082)$, and IL-10 $(P=0.894)$ genes.

\section{CORRELATION ANALYSIS}

The relationships between TLR expression and the relative abundance of epimural bacteria and cytokine expression are shown in Figure 3. The data revealed that the abundance levels of 11 taxa were correlated $(P<0.05)$ with the relative mRNA expression levels of TLR2 [six negative (unclassified Mollicutes, unclassified Coriobacteriaceae, unclassified Erysipelotrichaceae, unclassified Bacteria, Howardella, and unclassified Ruminococcaceae) and five positive (Ruminococcus, Butyrivibrio, Mogibacterium, unclassified Prevotellaceae, and Succiniclasticum)]; the abundance levels of 10 taxa were correlated $(P<0.05)$ with the relative mRNA expression levels of TLR5 [five negative (unclassified Mollicutes, unclassified Coriobacteriaceae, unclassified Erysipelotrichaceae, unclassified Bacteria, and unclassified Ruminococcaceae) and five positive (Ruminococcus, Butyrivibrio, Mogibacterium, unclassified Prevotellaceae, and Succiniclasticum)]; the abundance levels of eight taxa were correlated $(P<0.05)$ with the relative mRNA expression levels of TLR3 [five negative (unclassified Mollicutes, unclassified Erysipelotrichaceae, unclassified Rikenellaceae, Howardella, and unclassified Ruminococcaceae) and three positive (unclassified Clostridiales, Ruminococcus, and Butyrivibrio)]; the abundance of one taxa (unclassified Anaerolineaceae) was negatively associated $(P<0.05)$ with the relative mRNA expression of TLR4. In addition, the relative mRNA 
Table 2 | Effects of high-grain (HG) feeding on average relative abundance of genus level (\% of total sequences) in rumen epithelium, ranked by alphabetical order of first letter of phylum, family and genus name.

\begin{tabular}{|c|c|c|c|c|c|}
\hline Phylum & Family & Genus & \multicolumn{2}{|c|}{ Abundance (\%) } & $P$-value \\
\hline Actinobacteria & Coriobacteriaceae & Unclassified Coriobacteriaceae & $3.10 \pm 0.54$ & $0.86 \pm 0.22$ & 0.008 \\
\hline \multirow[t]{3}{*}{ Bacteroidetes } & Prevotellaceae & Prevotella & $4.36 \pm 0.79$ & $3.66 \pm 0.50$ & 0.548 \\
\hline & & Unclassified Prevotellaceae & $5.34 \pm 0.39$ & $8.30 \pm 0.80$ & 0.056 \\
\hline & Rikenellaceae & Unclassified Rikenellaceae & $5.80 \pm 0.56$ & $3.59 \pm 0.50$ & 0.016 \\
\hline \multirow[t]{8}{*}{ Firmicutes } & Lachnospiraceae & Acetitomaculum & $2.23 \pm 0.34$ & $1.33 \pm 0.29$ & 0.095 \\
\hline & & Butyrivibrio & $4.48 \pm 0.43$ & $17.55 \pm 2.05$ & 0.008 \\
\hline & & Howardella & $3.68 \pm 0.53$ & $1.75 \pm 0.23$ & 0.008 \\
\hline & & Syntrophococcus & $1.71 \pm 0.34$ & $1.13 \pm 0.08$ & 0.151 \\
\hline & & Unclassified Lachnospiraceae & $9.82 \pm 0.77$ & $9.50 \pm 0.78$ & 1.000 \\
\hline & Ruminococcaceae & Ruminococcus & $0.45 \pm 0.05$ & $2.20 \pm 0.52$ & 0.008 \\
\hline & Veillonellaceae & Succiniclasticum & $1.16 \pm 0.22$ & $3.21 \pm 0.35$ & 0.008 \\
\hline & Erysipelotrichaceae & Unclassified Erysipelotrichaceae & $5.08 \pm 0.46$ & $2.76 \pm 0.31$ & 0.008 \\
\hline \multirow[t]{4}{*}{ Proteobacteria } & Comamonadaceae & Comamonas & $2.59 \pm 0.37$ & $2.69 \pm 0.34$ & 1.000 \\
\hline & Neisseriaceae & Unclassified Neisseriaceae & $4.57 \pm 2.10$ & $0.14 \pm 0.05$ & 0.008 \\
\hline & Desulfobulbaceae & Desulfobulbus & $5.74 \pm 1.26$ & $5.27 \pm 0.63$ & 1.000 \\
\hline & Campylobacteraceae & Campylobacter & $2.27 \pm 0.23$ & $1.96 \pm 0.48$ & 0.841 \\
\hline Tenericutes & Unclassified Mollicutes & Unclassified Mollicutes & $2.27 \pm 0.21$ & $0.30 \pm 0.07$ & 0.008 \\
\hline
\end{tabular}

Only results obtained for the predominant bacterial genera (relative abundance $>1 \%$ in at least one sample) are presented. Values shown are means \pm SEM, $n=5$.

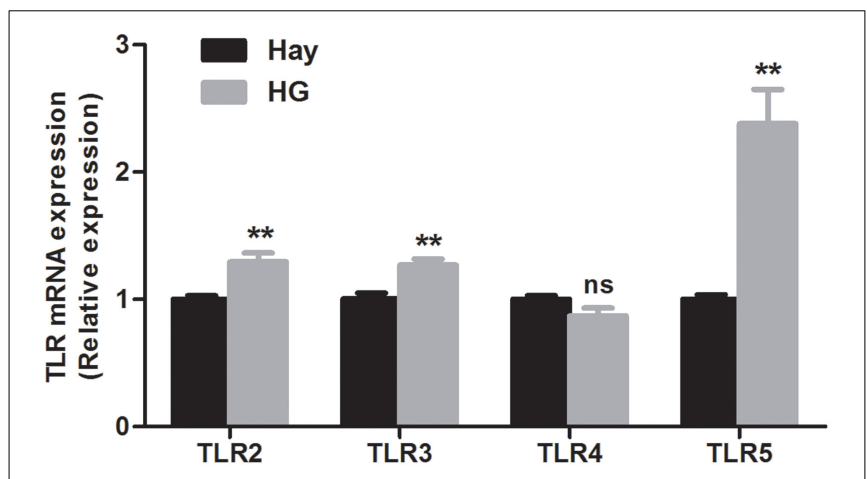

FIGURE 2 | Effects of high-grain feeding on the relative mRNA expression of Toll-like receptors in the rumen epithelium of goats (means \pm SEM, $\boldsymbol{n}=\mathbf{5}$ ). All analyses were performed in triplicate. ${ }^{* *} P<0.01$, NS: not significant.

levels of cytokines IL- $1 \beta$, IL- 6 , and IFN- $\gamma$ were positively associated with the relative mRNA expression levels of TLR2 and TLR3 $(P<0.05)$; the relative mRNA expression of cytokine IFN- $\gamma$ was positively correlated with TLR5 expression $(P<0.05)$; and TNF$\alpha$ mRNA expression was negatively associated with TLR4 mRNA expression $(P<0.05)$.

\section{DISCUSSION}

At the phylum level, Firmicutes (53.12\%) were the most abundant bacteria detected in the epimural bacterial community in the goats examined in this study, followed by Bacteroidetes (20.88\%) and Proteobacteria (13.35\%). This finding confirmed the findings reported in previous studies that phylum Firmicutes are relatively common in tissue-adherent populations (Sadet-Bourgeteau et al., 2010; Chen et al., 2011; Petri et al., 2013). In addition, the relative abundance of phylum Proteobacteria was higher than that in rumen content (the relative abundance of phylum Proteobacteria was $0.54 \%$ in rumen content; unpublished data), which was consistent with previous studies on sheep and heifers (Chen et al., 2011; Petri et al., 2013). The greater abundance of phylum Proteobacteria in the rumen epithelium was believed to be caused by the trace amounts of oxygen diffused through the tissue, which might favor a higher density of Proteobacteria, as many members of this phylum are microaerophiles or facultative anaerobes, and hence, not sensitive to oxygen toxicity (Sadet-Bourgeteau et al., 2010).

It is well known that diet influences ruminal fermentation characteristics and the structure of content-associated rumen microbial population (Russell and Rychlik, 2001; Sadet et al., 2007; Hook et al., 2011; Huo et al., 2014). However, the effects of diet on the epimural bacterial community remain controversial. A previous study, using PCR-DGGE technology, reported that the epimural community in lambs was less influenced by diet than were the microbiota associated with rumen contents (Sadet et al., 2007). Using PCR-DGGE and qRT- PCR analysis, Chen et al. (2011) demonstrated that an HG diet affects the diversity, but not density, of epimural bacteria in heifers. Petri et al. (2013) demonstrated, through pyrosequencing, that less than $5 \%$ of the 


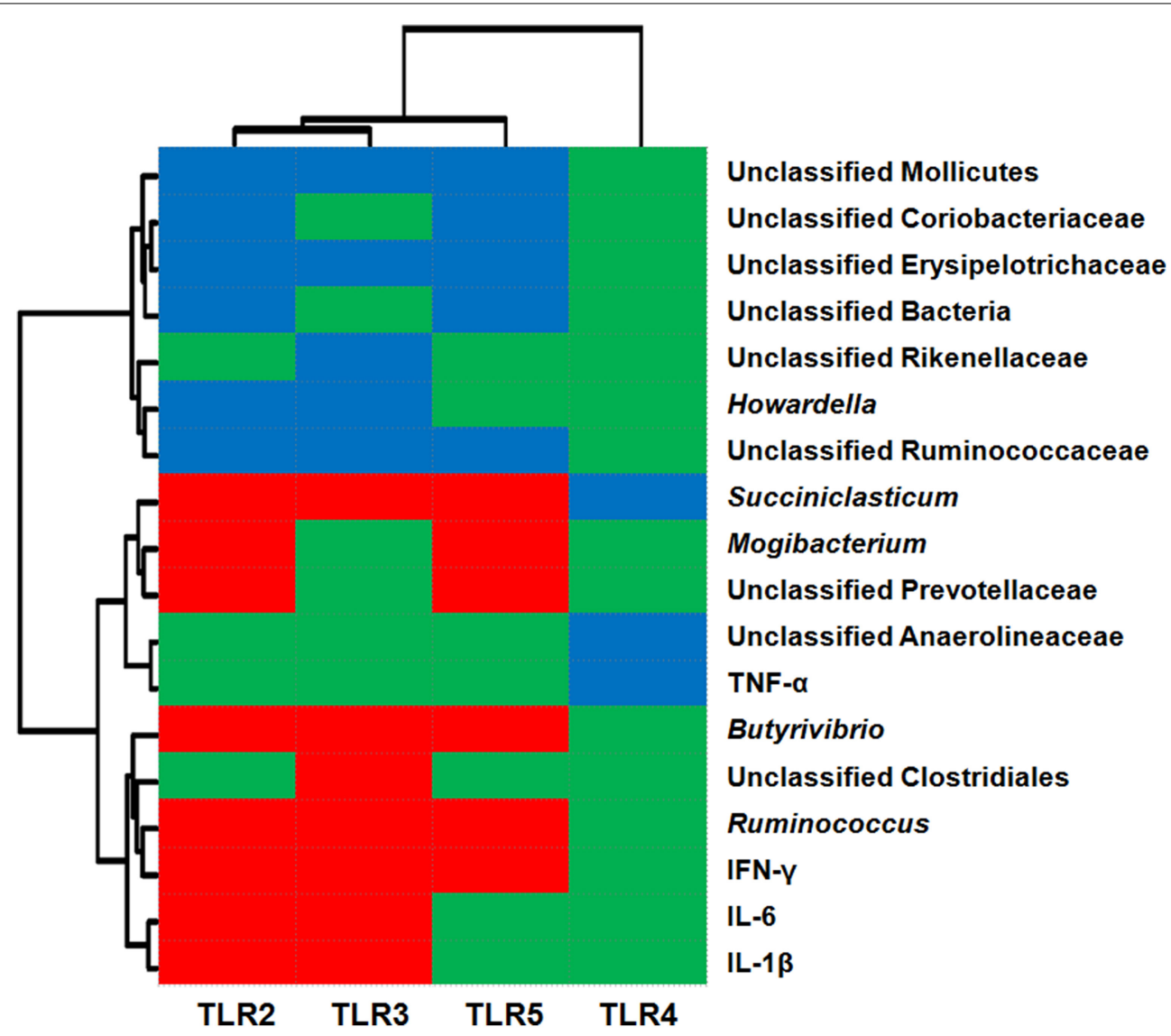

FIGURE 3 | Correlation analysis between the relative mRNA expression of Toll-like receptors (TLRs) and relative abundance of epithelium-associated microbiota (at the genus level) and mRNA expression of cytokines. Only results obtained for the predominant bacterial genera (relative abundance $>1 \%$ in at least one sample) for which the abundance was significantly associated with TLRs are shown. Cells are colored based on Pearson's correlation coefficient. Red represents a significant positive correlation $(P<0.05)$, blue represents a significant negative correlation $(P<0.05)$, and green represents a non-significant correlation $(P>0.05)$. total OTUs identified exhibited significant variability across diets in heifers. In the present study, the data revealed that HG feeding decreased epimural bacterial diversity and caused a strong shift in bacterial structure and composition. Furthermore, the Venn profile indicated that there were 299 unique OTUs belonging to the hay-fed goats and 227 unique OTUs belonging to the HG-fed goats. These results, which provide evidence that HG feeding influences the epimural bacterial community, are somewhat inconsistent with those of previous studies (Chen et al., 2011; Petri et al., 2013).

There are several possible reasons for the discrepancies between our results and those of previous studies. First, the differences in ruminant species might contribute to the variations in the epimural bacterial changes detected. Because the epimural bacteria inhabit the host tissues, it is suggested that the host species might play a role in regulating bacterial diversity and density. In the previous studies, the strong host effect might have masked the diet effect in sheep and heifers (Sadet et al., 2007; Sadet-Bourgeteau et al., 2010; Chen et al., 2011). The individual difference of goats was smaller than that of sheep and cattle (Liu et al., 2013), and thus, the diet effect masked by the host effect might be smaller in the present study. Second, variations in the type of grain in the diet might also have resulted in the differences in epimural bacterial response. Wheat was grain component for the sheep study (Sadet-Bourgeteau et al., 2010) and barley used as the concentrated diet in the heifer studies (Chen et al., 2011; Petri et al., 2013), while corn and wheat were the grain components in our present study. The differences in ruminal degradation of these cereal grains might contribute to the variations in epimural bacterial response. Third, and perhaps most important, we must take into consideration the HG-induced changes in rumen epithelial structure when comparing our results to those of Petri et al. (2013). Our previous study demonstrated that the HG diet used in this study caused parakeratosis, budding, and extensive sloughing of the stratum corneum (Liu et al., 2013), which potentially increases the availability of attachment sites for opportunistic bacteria (Plaizier et al., 2012) (Supplementary Figure $5)$. In addition, many previous studies have demonstrated that parakeratosis is linked with quantitative changes in the epimural microbial flora (Semjen et al., 1982; Steele et al., 2009). Thus, disruption of the rumen epithelial structure might account for the increased effect in the present study. Finally, the differences 
in analysis techniques, DNA extraction methods and sampling methods between our study and previous studies may also play a role in the discrepancies.

Most of the effects of the HG diet were observed at the genus level. The present study found that an HG feeding supports a higher proportion of members of several genera in the rumen epithelium, including Butyrivibrio, Succiniclasticum, Mogibacterium, and Ruminococcus (Table 2). The effect of the HG diet on the abundance of Succiniclasticum, Mogibacterium, and Ruminococcus was consistent with the results of Petri et al. (2013). However, the effect of HG feeding on the abundance of Butyrivibrio was inconsistent with the results of that study, in which the dietary change from a low-grain to an HG diet were much larger than ours (Petri et al., 2013). Those researchers found that HG feeding decreased the relative abundance of Butyrivibrio fibrisolvens in the rumen epithelium. This discrepancy might be associated with the differences in diet formulation and ruminant species, as mentioned earlier. Also, previous study demonstrated many Butyrivibrio species are amylolytic (Cotta, 1988), which may be an important potential reason for the increase in the abundance of rumen epithelial Butyrivibrio during $\mathrm{HG}$ feeding in the present study. Furthermore, as epithelial Butyrivibrio release butyrate close to the epithelium, they might enhance butyrate bioavailability for the host, which is useful for rumen epithelial proliferation (Sakata and Tamate, 1978; Shen et al., 2004). Therefore, an increase in the relative abundance of Butyrivibrio in the rumen epithelium might be an adaptation response to repair a damaged rumen epithelium by increasing epithelial proliferation during long-term HG feeding. Mogibacterium are anaerobic, Gram-positive, non-fermentative bacteria, first identified in the rumen epithelium by Li et al. (2012); the function of this genus in the rumen epithelium is unknown. Many previous studies have reported that this genus was commonly associated with periodontal disease and infected root canals in the human mouth (Nakazawa et al., 2000; Sato et al., 2012). Furthermore, Chen et al. (2012) reported that Mogibacterium was enriched in the colonic mucosa of colorectal cancer patients. Thus, these findings imply that the increase in the abundance of Mogibacterium during HG feeding could have some deleterious effects on rumen epithelial health. Ruminococcus are Gram-positive bacteria that were once considered to be cellulose-degrading bacteria in rumen content. Some Ruminococcus species are able to degrade mucin and are linked to gastrointestinal disease in humans (Crost et al., 2013; Wang et al., 2013). However, the physiological roles of Ruminococcus associated with rumen epithelium are unknown. This indicates the need for more research on the interactions between rumen epimural bacterial community and rumen epithelium function.

It is well known that TLRs can recognize the sensing of host epimural bacteria and bacterial products and trigger immune responses critical for maintaining host-microbial homeostasis in monogastric animals (Hooper et al., 2012). Dysregulation of this process can result in chronic inflammation of the gastrointestinal tract (Abreu, 2010). In addition to reporting changes in the bacterial composition of the rumen epithelium, this study, for the first time, report changes in the mRNA expression of TLRs in the rumen epithelium of goats during HG feeding.
Although the function of these epimural bacteria is not yet fully understood (Chen et al., 2012), the observed correlations between relative abundance of epimural bacteria and expression of the TLR genes support our speculation that epimural bacteria play a role in stimulating the innate immune response of the rumen epithelium in goats. Our study demonstrated that the HG diet increased the relative expression levels of TLR2, TLR3, and TLR5. A previous study indicated that TLR-2 is mainly involved in responses to cell wall components of Gram-positive bacteria (Melmed et al., 2003). Therefore, the increased expression of TLR2 might be dependent on the overgrowth of Grampositive bacteria Butyrivibrio, Succiniclasticum, Mogibacterium, and Ruminococcus members of the Firmicutes. TLR-5 has a role in the recognition of bacterial flagellin (Rhee et al., 2005; Abreu, 2010). Thus, the observed upregulation of TLR5 expression might be dependent on the overgrowth of bacteria rich in flagellin, such as Butyrivibrio. Furthermore, many studies on humans have indicated that TLR5 is expressed only on the basolateral surface, where it can trigger the production of cytokines in response to basolateral flagellin (Abreu, 2010). Another study demonstrated that luminal flagellin was only able to activate TLR5 after injury to the epithelial barrier (Rhee et al., 2005). Thus, the epithelial barrier also determines whether the bacteria and their products access the basolateral surface and trigger TLR5 expression. In our previous study, the HG diet increased rumen epithelial permeability (Liu et al., 2013), allowing bacteria or bacterial products from the rumen lumen to enter the mucosal tissue, which can stimulate host immune responses. Our correlation results revealed that the relative mRNA expression of TLR5 was positively associated with IFN- $\gamma$ production. Therefore, the observed differences in TLR5 expression and antimicrobial defense molecules between the two diets might be a result of increased permeability that allows the exposure of pattern recognition receptors and secretory molecules to epimural bacteria. It has also been reported that altered epithelial permeability is associated with changes in gastrointestinal microbes, which can subsequently affect host metabolism and rumen health (Ulluwishewa et al., 2011).

It has been reported that epimural bacterial composition regulates the expression of epithelial TLRs (Hooper et al., 2012), which triggers the production of cytokines in the intestine of monogastrics (Abreu, 2010). If the results of studies on intestinal tissue can be applied to ruminal tissue, it seems that the increase in expression of cytokines, reported in our previous study (Liu et al., 2013), might be associated with the activation of TLRs during HG diet feeding in goats. Our correlation results also confirmed this inference. However, it is not conclusive whether variations of epimural bacterial community and host TLR genes are the factors that contribute to the initial development of local inflammation in the rumen epithelium during HG diet feeding. Indeed, a very recent study regarding expression of genes and proteins with key protection functions in the rumen epithelium of goats fed low and high grain revealed that a mismatch in gene and protein expression in response to grain level (Hollmann et al., 2013), indicating the upregulation in the expression of TLR genes may not result in an increase in the expression of corresponding proteins. In addition, a previous study showed that a certain degree of upregulation of TLR genes may activate the mucosal innate system in 
rumen epithelium (Chen et al., 2012), implying the upregulation of TLR gene may be not a negative event for the host and may have a positive effect in preventing damages to the epithelial tissues at some condition. Thus, future studies are needed to determine whether variations in epimural bacterial community are associated with changes in protein expression of host TLRs during HG feeding, as well as how TLRs shape host immune response.

\section{CONCLUSION}

In general, although a relatively low number of animals were used in the current study, we observed that HG diet feeding causes a strong shift in the epimural bacterial community, and that these changes are associated with alterations in the relative expression of TLRs in the rumen epithelium. This study provides a first insight into the adaptive response of epimural bacterial populations to $\mathrm{HG}$ feeding in goats, using the pyrosequencing technique, and shows that these changes were associated with alterations in TLR expression. These findings lead to new insight into host-microbial relationships in ruminants and provide new opportunities to improve ruminant health under the current intensive production system.

\section{AUTHOR CONTRIBUTIONS}

JL carried out the majority of the experiment including animal care, VFA analysis, RNA isolation and real-time PCR; GB, JL and SM were responsible for pyrosequencing data processing, analysis and interpretation; SM and WZ contributed to the conception of the project; The manuscript was prepared by JL and SM.

\section{ACKNOWLEDGMENTS}

Funding support: National Natural Science Foundation of China (No. 31172228) and Jiangsu Planned Projects for Postdoctoral Research Funds (No. 1402149C).

\section{SUPPLEMENTARY MATERIAL}

The Supplementary Material for this article can be found online at: http://www.frontiersin.org/journal/10.3389/fmicb.2015.

\section{7/abstract}

\section{REFERENCES}

Abreu, M. T. (2010). Toll-like receptor signalling in the intestinal epithelium: how bacterial recognition shapes intestinal function. Nat. Rev. Immunol. 10, 131-144. doi: 10.1038/nri2707

Callaway, T. R., Dowd, S. E., Edrington, T. S., Anderson, R. C., Krueger, N., Bauer, N., et al. (2010). Evaluation of bacterial diversity in the rumen and feces of cattle fed different levels of dried distillers grains plus solubles using bacterial tag-encoded FLX amplicon pyrosequencing. J. Anim. Sci. 88, 3977-3983. doi: 10.2527/jas.2010-2900

Charavaryamath, C., Fries, P., Gomis, S., Bell, C., Doig, K., Guan, L. L., et al. (2011). Mucosal changes in a long-term bovine intestinal segment model following removal of ingesta and microflora. Gut Microbes 2, 134-144. doi: 10.4161/gmic.2.3.16483

Chen, W., Liu, F., Ling, Z., Tong, X., and Xiang, C. (2012). Human intestinal lumen and mucosa-associated microbiota in patients with colorectal cancer. PLOS ONE 7:e39743. doi: 10.1371/journal.pone.0039743

Chen, Y. H., Penner, G. B., Li, M. J., Oba, M., and Guan, L. L. (2011). Changes in bacterial diversity associated with epithelial tissue in the beef cow rumen during the transition to a high-grain diet. Appl. Environ. Microbiol. 77, 5770-5781. doi: 10.1128/AEM.00375-11

Cheng, K. J., Fay, J. P., Howarth, R. E., and Costerton, J. W. (1980). Sequence of events in the digestion of fresh legume leaves by rumen bacteria. Appl. Environ. Microbiol. 40, 613-625.
Cheng, K. J., and Wallace, R. J. (1979). The mechanism of passage of endogenous urea through the rumen wall and the role of ureolytic epithelial bacteria in the urea flux. Br. J. Nutr. 42, 553-557. doi: 10.1079/BJN19790147

Chomczynski, P., and Sacchi, N. (1987). Single-step method of RNA isolation by acid guanidinium thiocyanate-phenol-chloroform extraction. Anal. Biochem. 162, 156-159. doi: 10.1016/0003-2697(87)90021-2

Cotta, M. A. (1988). Amylolytic activity of selected species of ruminal bacteria. Appl. Environ. Microbiol. 54, 772-776.

Crost, E. H., Tailford, L. E., Le Gall, G., Fons, M., Henrissat, B., and Juge, N. (2013). Utilisation of mucin glycans by the human gut symbiont Ruminococcus gnavus is strain-dependent. PLOS ONE 8:e76341. doi: 10.1371/journal.pone.0076341

Dinsdale, D., Cheng, K. J., Wallace, R. J., and Goodlad, R. A. (1980). Digestion of epithelial tissue of the rumen wall by adherent bacteria in infused and conventionally fed sheep. Appl. Environ. Microbiol. 39, 1059-1066.

Graham, C., and Simmons, N. L. (2005). Functional organization of the bovine rumen epithelium. Am. J. Physiol. Regul. Integr. Comp. Physiol. 288, R173-R181. doi: 10.1152/ajpregu.00425.2004

Hollmann, M., Miller, I., Hummel, K., Sabitzer, S., Metzler-Zebeli, B. U., RazzaziFazeli, E., et al. (2013). Downregulation of cellular protective factors of rumen epithelium in goats fed high energy diet. PLoS ONE 8:e81602. doi: 10.1371/journal.pone.0081602

Hook, S. E., Steele, M. A., Northwood, K. S., Dijkstra, J., France, J., Wright, A. D., et al. (2011). Impact of subacute ruminal acidosis (SARA) adaptation and recovery on the density and diversity of bacteria in the rumen of dairy cows. FEMS Microbiol. Ecol. 78, 274-285. doi: 10.1111/j.1574-6941.2011.01154.x

Hooper, L. V., Littman, D. R., and Macpherson, A. J. (2012). Interactions between the microbiota and the immune system. Science 336, 1268-1273. doi: 10.1126/science. 1223490

Huo, W., Zhu, W., and Mao, S. (2014). Impact of subacute ruminal acidosis on the diversity of liquid and solid-associated bacteria in the rumen of goats. World J. Microb. Biot. 30, 669-680. doi: 10.1007/s11274-013-1489-8

Li, M. J., Zhou, M., Adamowicz, E., Basarab, J. A., and Guan, L. L. (2012). Characterization of bovine ruminal epithelial bacterial communities using $16 \mathrm{~S}$ rRNA sequencing, PCR-DGGE, and qRT-PCR analysis. Vet. Microbiol. 155, 72-80. doi: 10.1016/j.vetmic.2011.08.007

Liu, J. H., Xu, T. T., Liu, Y. J., Zhu, W. Y., and Mao, S. Y. (2013). A highgrain diet causes massive disruption of ruminal epithelial tight junctions in goats. Am. J. Physiol. Regul. Integr. Comp. Physiol. 305, R232-R241. doi: 10.1152/ajpregu.00068.2013

Lozupone, C., and Knight, R. (2005). UniFrac: a new phylogenetic method for comparing microbial communities. Appl. Environ. Microbiol. 71, 8228-8235. doi: 10.1128/AEM.71.12.8228-8235.2005

Malmuthuge, N., Li, M., Fries, P., Griebel, P. J., and Guan, L. L. (2012). Regional and age dependent changes in gene expression of Toll-like receptors and key antimicrobial defence molecules throughout the gastrointestinal tract of dairy calves. Vet. Immunol. Immunopathol. 146, 18-26. doi: 10.1016/j.vetimm.2012.01.010

McCowan, R. P., Cheng, K. J., Bailey, C. B., and Costerton, J. W. (1978). Adhesion of bacteria to epithelial cell surfaces within the reticulo-rumen of cattle. Appl. Environ. Microbiol. 35, 149-155.

McCowan, R. P., Cheng, K. J., and Costerton, J. W. (1980). Adherent bacterial populations on the bovine rumen wall: distribution patterns of adherent bacteria. Appl. Environ. Microbiol. 39, 233-241.

Melmed, G., Thomas, L. S., Lee, N., Tesfay, S. Y., Lukasek, K., Michelsen, K. S., et al. (2003). Human intestinal epithelial cells are broadly unresponsive to Toll-like receptor 2-dependent bacterial ligands: implications for host-microbial interactions in the gut. J. Immunol. 170, 1406-1415. doi: 10.4049/jimmunol.170.3.1406

Metzler-Zebeli, B. U., Schmitz-Esser, S., Klevenhusen, F., Podstatzky-Lichtenstein, L., Wagner, M., and Zebeli, Q. (2013). Grain-rich diets differently alter ruminal and colonic abundance of microbial populations and lipopolysaccharide in goats. Anaerobe 20, 65-73. doi: 10.1016/j.anaerobe.2013.02.005

Nakazawa, F., Sato, M., Poco, S. E., Hashimura, T., Ikeda, T., Kalfas, S., et al. (2000). Description of Mogibacterium pumilum gen. nov., sp. nov. and Mogibacterium vescum gen. nov., sp. nov., and reclassification of Eubacterium timidum (Holdeman et al. 1980) as Mogibacterium timidum gen. nov., comb. nov. Int. J. Syst. Evol. Microbiol. 50(Pt 2), 679-688. doi: 10.1099/00207713-50-2-679

Petri, R. M., Schwaiger, T., Penner, G. B., Beauchemin, K. A., Forster, R. J., McKinnon, J. J., et al. (2013). Changes in the rumen epimural bacterial diversity of beef cattle as affected by diet and induced ruminal acidosis. Appl. Environ. Microbiol. 79, 3744-3755. doi: 10.1128/AEM.03983-12 
Plaizier, J. C., Khafipour, E., Li, S., Gozho, G. N., and Krause, D. O. (2012). Subacute ruminal acidosis (SARA), endotoxins and health consequences. Anim. Feed Sci. Technol. 172, 9-21. doi: 10.1016/j.anifeedsci.2011.12.004

Pruesse, E., Quast, C., Knittel, K., Fuchs, B. M., Ludwig, W., Peplies, J., et al. (2007). SILVA: a comprehensive online resource for quality checked and aligned ribosomal RNA sequence data compatible with ARB. Nuc. Acid. Res. 35, 7188-7196. doi: 10.1093/nar/gkm864

Quince, C., Lanzen, A., Davenport, R. J., and Turnbaugh, P. J. (2011). Removing noise from pyrosequenced amplicons. BMC Bioinformatics 12:38. doi: 10.1186/1471-2105-12-38

Rhee, S. H., Im, E., Riegler, M., Kokkotou, E., O’Brien, M., and Pothoulakis, C. (2005). Pathophysiological role of Toll-like receptor 5 engagement by bacterial flagellin in colonic inflammation. Proc. Natl. Acad. Sci. U.S.A. 102, 13610-13615. doi: 10.1073/pnas.0502174102

Russell, J. B., and Rychlik, J. L. (2001). Factors that alter rumen microbial ecology. Science 292, 1119-1122. doi: 10.1126/science. 1058830

Sadet, S., Martin, C., Meunier, B., and Morgavi, D. P. (2007). PCR-DGGE analysis reveals a distinct diversity in the bacterial population attached to the rumen epithelium. Animal 1, 939-944. doi: 10.1017/S17517311070 00304

Sadet-Bourgeteau, S., Martin, C., and Morgavi, D. P. (2010). Bacterial diversity dynamics in rumen epithelium of wethers fed forage and mixed concentrate forage diets. Vet. Microbiol. 146, 98-104. doi: 10.1016/j.vetmic.2010. 04.029

Sakata, T., and Tamate, H. (1978). Rumen epithelial cell proliferation accelerated by rapid increase in intraruminal butyrate. J. Dairy Sci. 61, 1109-1113. doi: 10.3168/jds.S0022-0302(78)83694-7

Sato, T., Yamaki, K., Ishida, N., Hashimoto, K., Takeuchi, Y., Shoji, M., et al. (2012). Cultivable anaerobic microbiota of infected root canals. Int. J. Dent. 2012:609689. doi: 10.1155/2012/609689

Schloss, P. D., Gevers, D., and Westcott, S. L. (2011). Reducing the effects of PCR amplification and sequencing artifacts on 16S rRNA-based studies. PLoS ONE 6:e27310. doi: 10.1371/journal.pone.0027310

Schloss, P. D., Westcott, S. L., Ryabin, T., Hall, J. R., Hartmann, M., Hollister, E. B., et al. (2009). Introducing mothur: open-source, platform-independent, community-supported software for describing and comparing microbial communities. Appl. Environ. Microbiol. 75, 7537-7541. doi: 10.1128/AEM. 01541-09

Semjen, G., Galfi, P., and Kutas, F. (1982). Effect of parakeratosis on bacterial adherence to ruminal epithelium. Zentralbl. Veterinarmed. B 29, 317-319. doi: 10.1111/j.1439-0450.1982.tb01231.x

Shen, Z., Seyfert, H. M., Lohrke, B., Schneider, F., Zitnan, R., Chudy, A., et al. (2004). An energy-rich diet causes rumen papillae proliferation associated with more IGF type 1 receptors and increased plasma IGF-1 concentrations in young goats. J. Nutr. 134, 11-17.
Steele, M. A., Hook, S. E., Alzahal, O., Croom, W. J., and McBride, B. W. (2009). Morphologic adaptations of the rumen epithelium during subacute ruminal acidosis. Can. J. Ani. Sci. 89, 141-142.

Sun, Y. Z., Mao, S. Y., Yao, W., and Zhu, W. Y. (2008). DGGE and 16S rDNA analysis reveals a highly diverse and rapidly colonising bacterial community on different substrates in the rumen of goats. Animal 2, 391-398. doi: $10.1017 /$ S1751731107001231

Ulluwishewa, D., Anderson, R. C., McNabb, W. C., Moughan, P. J., Wells, J. M., and Roy, N. C. (2011). Regulation of tight junction permeability by intestinal bacteria and dietary components. J. Nutr. 141, 769-776. doi: 10.3945/jn.110.135657

Wallace, R. J., Cheng, K. J., Dinsdale, D., and Orskov, E. R. (1979). An independent microbial flora of the epithelium and its role in the ecomicrobiology of the rumen. Nature 279, 424-426. doi: 10.1038/279424a0

Wang, A., Gu, Z., Heid, B., Akers, R. M., and Jiang, H. (2009). Identification and characterization of the bovine $\mathrm{G}$ protein-coupled receptor GPR41 and GPR43 genes. J. Dairy Sci. 92, 2696-2705. doi: 10.3168/jds.2009-2037

Wang, L., Christophersen, C. T., Sorich, M. J., Gerber, J. P., Angley, M. T., and Conlon, M. A. (2013). Increased abundance of Sutterella spp. and Ruminococcus torques in feces of children with autism spectrum disorder. Mol. Autism. 4:42. doi: 10.1186/2040-2392-4-42

Wang, Q., Garrity, G. M., Tiedje, J. M., and Cole, J. R. (2007). Naive Bayesian classifier for rapid assignment of rRNA sequences into the new bacterial taxonomy. Appl. Environ. Microbiol. 73, 5261-5267. doi: 10.1128/AEM.00062-07

Zened, A., Combes, S., Cauquil, L., Mariette, J., Klopp, C., Bouchez, O., et al. (2013). Microbial ecology of the rumen evaluated by 454 GS FLX pyrosequencing is affected by starch and oil supplementation of diets. FEMS Microbiol. Ecol. 83, 504-514. doi: 10.1111/1574-6941.12011

Conflict of Interest Statement: The authors declare that the research was conducted in the absence of any commercial or financial relationships that could be construed as a potential conflict of interest.

Received: 01 December 2014; accepted: 12 February 2015; published online: 02 March 2015.

Citation: Liu J, Bian G, Zhu W and Mao S (2015) High-grain feeding causes strong shifts in ruminal epithelial bacterial community and expression of Toll-like receptor genes in goats. Front. Microbiol. 6:167. doi: 10.3389/fmicb.2015.00167

This article was submitted to Microbial Immunology, a section of the journal Frontiers in Microbiology.

Copyright (c) 2015 Liu, Bian, Zhu and Mao. This is an open-access article distributed under the terms of the Creative Commons Attribution License (CC BY). The use, distribution or reproduction in other forums is permitted, provided the original author(s) or licensor are credited and that the original publication in this journal is cited, in accordance with accepted academic practice. No use, distribution or reproduction is permitted which does not comply with these terms. 